\title{
Distinct Neuroanatomical Correlates of Neuropsychiatric Symptoms in the Three Main Forms of Genetic Frontotemporal Dementia in the GENFI Cohort
}

Leila Sellami $^{\mathrm{m}, 1}$, Martina Bocchetta ${ }^{\mathrm{a}, 1}$, Mario Masellis ${ }^{\mathrm{f}}$, David M. Cash $^{\mathrm{a}, \mathrm{b}}$, Katrina M. Dick ${ }^{\mathrm{a}}$, John van Swieten ${ }^{\mathrm{c}}$, Barbara Borroni ${ }^{\mathrm{d}}$, Daniela Galimberti ${ }^{\mathrm{e}}$, Maria Carmela Tartaglia ${ }^{\mathrm{g}}$, James B. Rowe ${ }^{\mathrm{h}}$, Caroline Graff ${ }^{\mathrm{i}, \mathrm{j}}$, Fabrizio Tagliavini ${ }^{\mathrm{k}}$, Giovanni Frisoni ${ }^{\mathrm{l}}$, Elizabeth Finger ${ }^{\mathrm{n}}$, Alexandre de Mendonça ${ }^{\mathrm{o}}$, Sandro Sorbi ${ }^{\mathrm{p}, \mathrm{q}}$, Jason D. Warren ${ }^{\mathrm{a}}$, Jonathan D. Rohrer ${ }^{\mathrm{a}, 2}$ and Robert Jr. Laforce ${ }^{\mathrm{m}, 2, *}$ on behalf of the Genetic FTD Initiative, GENFI ${ }^{3}$

${ }^{a}$ Department of Neurodegenerative Disease, Dementia Research Centre, UCL Institute of Neurology, Queen Square, London, UK

${ }^{\mathrm{b}}$ Centre for Medical Image Computing, UCL, UK

${ }^{\mathrm{c}}$ Erasmus Medical Center, Rotterdam, Netherlands

${ }^{\mathrm{d}}$ University of Brescia, Brescia, Italy

e Department of Pathophysiology and Transplantation, "Dino Ferrari” Center, University of Milan, Fondazione Cá Granda, IRCCS Ospedale Maggiore Policlinico, Milan, Italy

${ }^{\mathrm{f}}$ Cognitive Neurology Research Unit, Sunnybrook Health Sciences Centre; Hurvitz Brain Sciences Research Program, Sunnybrook Research Institute; Department of Medicine, University of Toronto, Toronto, ON, Canada ${ }^{\mathrm{g}}$ Tanz Centre for Research in Neurodegenerative Diseases, University of Toronto, Toronto, ON, Canada

${ }^{\mathrm{h}}$ University of Cambridge, Cambridge, $U K$

${ }^{\mathrm{i}}$ Karolinska Institutet, Stockholm, Sweden; Department NVS, Center for Alzheimer Research, Division of Neurogeriatrics, Sweden

${ }^{\mathrm{j} D e p a r t m e n t ~ o f ~ G e r i a t r i c ~ M e d i c i n e, ~ K a r o l i n s k a ~ U n i v e r s i t y ~ H o s p i t a l, ~ S t o c k h o l m, ~ S w e d e n ~}$

${ }^{\mathrm{k}}$ Istituto Neurologico Carlo Besta, Milan, Italy

${ }^{1}$ IRCCS San Giovanni di Dio Fatebenefratelli Brescia, Italy

${ }^{\mathrm{m}}$ Clinique Interdisciplinaire de Mémoire (CIME), Université Laval, QC, Canada

${ }^{\mathrm{n}}$ University of Western Ontario, ON, Canada

${ }^{\circ}$ Faculdade de Medicina, Universidade de Lisboa, Portugal

${ }^{\mathrm{p}}$ Department of Neurosciences, Psychology, Drug Research and Child Health (NEUROFARBA), University of Florence, Florence, Italy

${ }^{\mathrm{q}}$ IRCCS Don Carlo Gnocchi, Florence, Italy

\footnotetext{
${ }^{1}$ These authors contributed equally to this work.

${ }^{2}$ Joint senior authors.

${ }^{3}$ List of consortium members in Supplementary Material.

*Correspondence to: Dr. Robert Jr. Laforce, Clinique Interdisciplinaire de Mémoire (CIME), Département des Sciences
}

Neurologiques, CHU de Québec; Faculté de médecine, Université Laval, Québec, Canada. 1401, 18ième rue, Québec G1J 1Z4, Canada. Tel.: +1 418649 5980; Fax: +1 418649 5981; E-mail: robert.laforce@fmed.ulaval.ca. 


\begin{abstract}
.
Background: The overlap between frontotemporal dementia (FTD) and primary psychiatric disorders has been brought to light by reports of prominent neuropsychiatric symptoms (NPS) in FTD-related genetic mutations, particularly among C9orf72 and GRN carriers. It has been recently demonstrated that early neuroanatomical changes in genetic FTD may be different across the major disease-causing mutations.
\end{abstract}

Objective: We aimed to identify whether NPS could be driven by distinct structural correlates.

Methods: One hundred and sixty-seven mutation carriers ( $75 \mathrm{GRN}, 60 \mathrm{C} 9 \mathrm{orf} 72$, and $32 \mathrm{MAPT}$ ) were included from the Genetic FTD Initiative (GENFI) study, a large international cohort of genetic FTD. Neuropsychiatric symptoms including delusions, hallucinations (visual, auditory, and tactile), depression, and anxiety were investigated using a structured interview. Voxel-based morphometry was performed to identify neuroanatomical correlates of NPS.

Results: Psychotic symptoms correlated mainly with grey matter (GM) atrophy in the anterior insula, left thalamus, cerebellum, and cortical regions including frontal, parietal, and occipital lobes in GRN mutations carriers. GM atrophy in posterior structures of the default-mode network was associated with anxiety in the GRN group. Delusions in C9orf72 expansion carriers were mainly associated with left frontal cortical atrophy. Cerebellar atrophy was found to be correlated only with anxiety in $C 9$ orf72 carriers. NPS in the MAPT group were mainly associated with volume loss in the temporal lobe.

Conclusion: Neuroanatomical correlates of NPS appear to be distinct across the main forms of genetic FTD. Overall, our findings support overlapping brain structural changes between FTD and primary psychiatric disorders.

Keywords: Frontotemporal dementia, genetics, magnetic resonance imaging, neuropsychiatry

\section{INTRODUCTION}

Frontotemporal dementia (FTD) is the second most common cause of young-onset dementia. It is a highly heritable disease with an autosomal dominant mode of inheritance being found in around a third of cases $[1,2]$. Mutations in three main genes have been identified in familial FTD: microtubule associated protein tau (MAPT), progranulin $(G R N)$, and chromosome 9 open reading frame 72 (C9orf72) [3]. Among neurodegenerative dementias, FTD often mimics primary psychiatric conditions. Indeed, patients with FTD are more likely to receive a prior inaccurate psychiatric diagnosis [4].

The most common subtype, namely behavioral variant FTD (bvFTD), is characterized by apathy, loss of empathy, perseverative and compulsive behavior, disinhibition, and hyperorality [5]. These symptoms often lead to bvFTD being misdiagnosed with either late onset depression or obsessive-compulsive and impulse-control disorders. Other neuropsychiatric symptoms (NPS) such as delusions or hallucinations are frequently present in FTD with a third of patients having psychotic symptoms in neuropathologically confirmed cases [6]. Although FTD may mimic late-onset schizophrenia or bipolar spectrum disorders in some cases, psychosis and mood disorders are not considered clinical hallmarks of bvFTD [7] and are not included within current diagnostic criteria.

Recent genetic advances in the understanding of familial forms of FTD have rekindled interest in the borderlands between FTD and psychiatric disorders, by revealing a high frequency of NPS in genetic FTD, particularly among $C 9$ orf 72 and $G R N$ mutation carriers [8-10]. NPS sometimes emerge many years prior to dementia onset and appear to have different phenomenology across FTD-related mutations [10, 11]. Cross sectional results from the Genetic FTD Initiative (GENFI) have previously shown evidence for neuroanatomical changes in presymptomatic FTD mutation carriers $[12,13]$. The insula has been shown to be the earliest affected region in genetic FTD, a key hub in the salience network which has been considered as an influential model in several psychiatric disorders such as schizophrenia [14]. To our knowledge, no study has been carried out to date, which assesses the correlation between NPS and neurostructural changes across the three main genes associated with FTD. Characterizing the neural correlates across familial FTD would provide important insight into the distinctive structures involved in the neuropsychiatric phenotype in FTD and therefore enable further understanding of the pathophysiological substrate and neurobiological basis of NPS. The pattern of brain atrophy could ultimately guide targeted genetic testing particularly in cases of atypical and overlapping FTD/neuropsychiatric presentations. In this study, we applied voxel-based morphometry (VBM) to a large international cohort of presymptomatic and symptomatic carriers of FTD-related mutations. We hypothesized that neuroanatomical correlates of NPS would be distinct between the different forms of genetic FTD and would bring evidence of overlapping 
brain structural changes with primary psychiatric disorders.

\section{MATERIALS AND METHODS}

\section{Participants}

We assessed the structural correlates of NPS in cases with genetic FTD enrolled within the GENFI cohort [12]. GENFI is a multicenter study, which during the first phase consisted of 13 research sites, in the UK, Italy, Portugal, the Netherlands, Sweden, and Canada. Participants recruited to GENFI were either known carriers of a pathogenic mutation in MAPT, GRN, or C9orf72, or at risk of carrying a mutation because a first-degree relative was a known symptomatic carrier. Between January 2012 and April 2015, 365 participants were recruited into GENFI, of which 205 were mutation carriers. Of the 205 mutation carriers, 167 participants had T1weighted MRI scans suitable for VBM analysis. All subjects underwent a standard clinical assessment consisting of a family history, past medical history, neuropsychological assessment, and physical examination. NPS including delusions, hallucinations, depression, and anxiety were also questioned. This was performed with the participant and carer, using a scale scored from 0 to 3 . The NPS scale used in the current study was adapted from the Neuropsychiatric Inventory [15]. The NPS scale includes 6 questions that cover the domains of psychosis and affective disorders. Each of the 6 individual symptom domains (visual, auditory and tactile hallucinations, delusions, depression, and anxiety) contains a survey question that reflects the main suggestive features. Initial answer to each domain is "present" or "absent". If "absent", the domain is scored 0. If "present", the severity is rated on a four-point scale $(0.5=$ questionable/very mild, $1=$ mild, $2=$ moderate and $3=$ severe). Both symptomatic and presymptomatic mutation carriers were included in the present study. In the context of this study, presymptomatic status refers to subjects who are carriers of a pathogenic mutation in MAPT, GRN, or C9orf72, without meeting the international consensus criteria for bvFTD, PPA or FTD-MND [5]. All participants from the GENFI cohort, that were assessed for neuropsychiatric symptoms and that had a suitable MRI scans for VBM, were included in the current study. Local ethics committees at each site approved the study and all participants provided written informed consent.

\section{MRI acquisition and VBM analysis}

Participants underwent volumetric T1-weighted MRI, according to the GENFI imaging protocol. Sites used different types of scanners: Siemens Trio 3T, Siemens Skyra 3T, Siemens 1.5T, Phillips 3T, General Electric (GE) 1.5T and GE 3T. Scan protocols were designed at the outset of the study to ensure adequate matching between scanners and image quality control. Voxel-based morphometry was performed using Statistical Parametric Mapping (SPM) 12 software, version 6906 (http://www.fil.ion.ucl.ac.uk/spm), running under Matlab R2012a (Math Works, USA). T1-weighted images were normalized and segmented into grey matter, white matter (WM), and cerebrospinal fluid (CSF) probability maps, by using standard procedures and the fast-diffeomorphic image registration algorithm (DARTEL) [16]. GM segments were affine transformed into the Montreal Neurological Institute (MNI) space, modulated and smoothed using a Gaussian kernel with $6 \mathrm{~mm}$ fullwidth at half maximum before analysis. Finally, a mask was applied as reported in [17]. Study-specific templates were created based on the subjects included in the specific analysis. At each stage, all segmentations were reviewed visually. Total intracranial volume (TIV) was calculated using SPM [18].

\section{Statistical analysis}

Differences in demographic and clinical characteristics were assessed using a Chi-squared test, and one-way ANOVA (with post hoc Tukey test) using SPSS software version 22 (SPSS Inc, Chicago, Illinois, USA). The significance threshold was set at 0.05. Correlations were calculated with the Spearman's rank coefficient $(\mathrm{rS})$.

Regarding the VBM analysis, the preprocessed GM tissue maps were fitted to a multiple regression model to identify the negative correlation between GM density and neuropsychiatric symptoms, which were measured by the NPS scale (score from 0 to $3)$. The analyses were run separately for each genetic group (GRN, MAPT, C9orf72) and for each symptom (visual hallucinations, auditory hallucinations, tactile hallucinations, delusions, depression and anxiety). Age, gender, TIV, and scanner type were entered as nuisance covariates. The uncorrected statistical threshold was set at $p<0.001$, with a minimum cluster size of 10 voxels. The Family-Wise Error (FWE) and False Discovery Rate (FDR) for multiple comparisons correction were set at 0.05 . 


\section{RESULTS}

Among the 167 mutation carriers, $45 \%$ had a mutation in the GRN gene, $36 \%$ in the C9orf 72 , and $19 \%$ in the MAPT. Participants consisted of 122 presymptomatic (63 GRN, 37 C9orf72, and 22 MAPT) and 45 symptomatic (12 GRN, 23 C9orf72, and 10 MAPT) carriers (see Table 1). Individuals with MAPT mutations were significantly younger (mean age 44.3 years versus 52.1 and 51.3 years in $C 9$ orf 72 and $G R N$, respectively; $p=0.013$ ). According to the Frontotemporal Dementia Rating Scale (FRS) [19], in the symptomatic group: three participants (1 GRN, $1 C 9$ orf72, and $1 M A P T)$ were mildly impaired, three (1 GRN, 1 C9orf72, and 1 MAPT) were moderately impaired, 18 (3 GRN, 12 C9orf72, and 3 MAPT) were severely impaired, 15 (5 GRN, 7 C9orf72, and 3 MAPT) were very severely impaired and $5(2$ $G R N, 1$ C9orf72, and $2 M A P T$ ) were profoundly impaired; FRS was not available for one patient. There was no significant correlation between mean NPS score and disease severity in symptomatic carriers $(\mathrm{rS}=0.21, p=0.15)$. There were no significant differences between the three genetic groups in terms of severity scores nor frequency of hallucinations (visual, auditory and tactile), delusions, depression and anxiety (see Table 2). Distribution of NPS among presymptomatic and symptomatic carriers is displayed in Supplementary Table 1.

Statistical parametric maps showing correlations between each NPS score and GM density in the three genetic groups are reported in Figs. 1 to 6. For visual hallucinations (Fig. 1), in the GRN group, we found significant negative correlations between the clinical score and GM in a large network of areas including mainly the parietal lobe (left superior parietal lobule and left angular gyrus), inferior occipital gyrus, left thalamus and left middle frontal gyrus (these clusters remained significant after FWE correction for multiple comparisons $p<0.05$ ), with further areas in the cerebellum and temporal lobe (Supplementary Table 2). In the C9orf72 group, we identified a significant negative correlation with GM in the parietal lobe (left superior parietal lobule and left angular gyrus) and left occipital pole (Supplementary Table 3). In the MAPT group, visual hallucinations score correlated with GM in the precentral gyrus, left lingual gyrus, right fusiform gyrus, and left angular gyrus (Supplementary Table 4). In the GRN group, auditory hallucinations (Fig. 2) were associated with the left

Table 1

Demographics

\begin{tabular}{lcccc}
\hline & $G R N(n=75)$ & C9orf72 $(n=60)$ & MAPT $(n=32)$ & $p$ \\
\hline Gender, female, n (\%) & $48(64 \%)$ & $31(51.7 \%)$ & $16(50 \%)$ & 0.24 \\
Right-handed, n (\%) & $66(88 \%)$ & $57(95 \%)$ & $29(90.6 \%)$ & 0.53 \\
Age (y) & $51.3(11.4)$ & $52.1(14.2)$ & $44.3(12)$ & $0.01^{*}$ \\
Education (y) & $13.4(3.5)$ & $13.5(3.7)$ & $12.9(3.8)$ & 0.75 \\
Clinical status & & & \\
$\quad$ Symptomatic, $\mathrm{n}(\%)$ & $12(16 \%)$ & $23(38 \%)$ & $10(31 \%)$ & 0.14 \\
Presymptomatic, $\mathrm{n}(\%)$ & $63(84 \%)$ & $37(62 \%)$ & $22(68 \%)$ & 0.48 \\
\hline
\end{tabular}

Data are No (\%) or mean (SD). *One-way ANOVA $p$ value $<0.05$, post hoc $p=0.027$ for MAPT versus $G R N$ and $p=0.015$ for $M A P T$ versus $C 90 r f 72$.

Table 2

Frequency and severity of neuropsychiatric symptoms (NPS) in the genetic groups

\begin{tabular}{llcccc}
\hline NPS & & GRN $(n=75)$ & C9orf72 $(n=60)$ & MAPT $(n=32)$ & $p$ \\
\hline Visual hallucinations & Frequency & $3(4 \%)$ & $4(7 \%)$ & $2(6 \%)$ & 0.77 \\
& Severity & $0.05(0.28 ; 0-2)$ & $0.15(0.57 ; 0-3)$ & $0.05(0.19 ; 0-1)$ & 0.31 \\
Auditory hallucinations & Frequency & $3(4 \%)$ & $3(5 \%)$ & $2(6 \%)$ & 0.88 \\
& Severity & $0.05(0.28 ; 0-2)$ & $0.09(0.46 ; 0-3)$ & $0.07(0.19 ; 0-1)$ & 0.76 \\
Tactile hallucinations & Frequency & $3(4 \%)$ & $2(3 \%)$ & $1(3 \%)$ & 0.96 \\
& Severity & $0.05(0.28 ; 0-2)$ & $0.04(0.26 ; 0-2)$ & $0.03(0.17 ; 0-1)$ & 0.91 \\
Delusions & Frequency & $4(5 \%)$ & $7(12 \%)$ & $3(9 \%)$ & 0.40 \\
& Severity & $0.07(0.30 ; 0-2)$ & $0.21(0.65 ; 0-3)$ & $0.13(0.42 ; 0-2)$ & 0.23 \\
Depression & Frequency & $16(21 \%)$ & $18(30 \%)$ & $6(19 \%)$ & 0.37 \\
& Severity & $0.24(0.52 ; 0-2)$ & $0.33(0.58 ; 0-2)$ & $0.23(0.55 ; 0-2)$ & 0.57 \\
Anxiety & Frequency & $12(16 \%)$ & $17(28 \%)$ & $9(28 \%)$ & 0.17 \\
& Severity & $0.18(0.46 ; 0-2)$ & $0.35(0.62 ; 0-2)$ & $0.34(0.64 ; 0-2)$ & 0.16 \\
\hline
\end{tabular}

Frequency is reported as No.(\%). Severity was assessed using a NPS Scale: $0=$ absent, $0.5=$ very mild, $1=$ mild, $2=$ moderate, $3=$ severe, data are mean score (SD and range are given in brackets). 

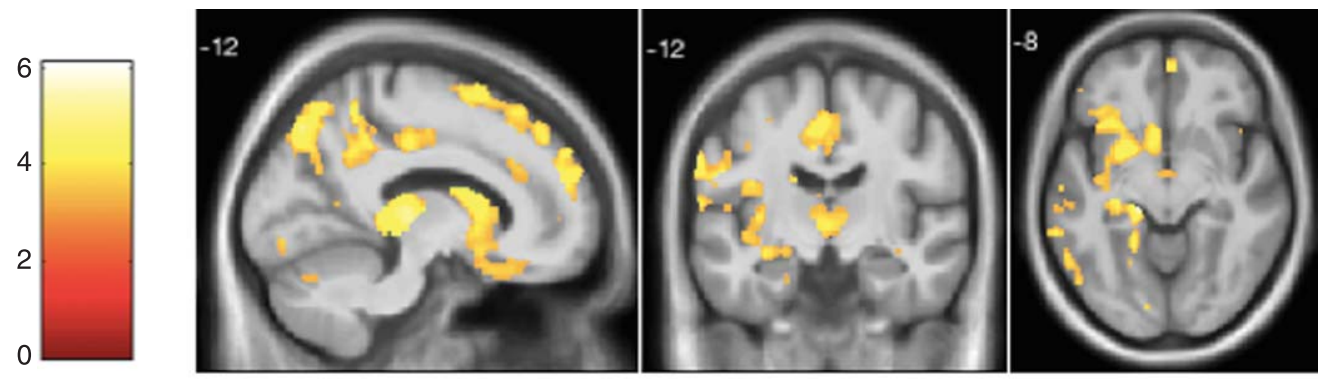

GRN
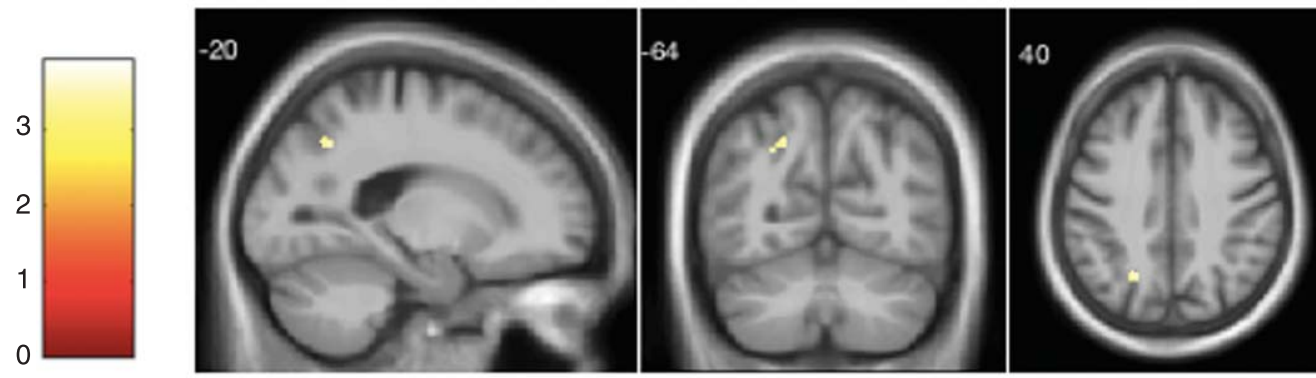

C9orf72
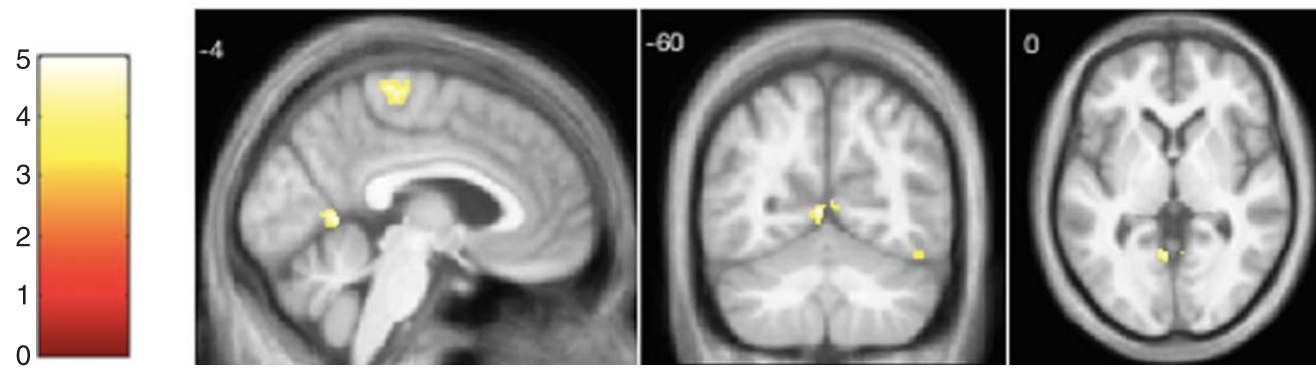

MAPT

Fig. 1. VBM analysis showing areas of significant correlation between the presence and severity of visual hallucinations and GM density across the FTD genetic groups. Statistical parametric maps were thresholded at $p<0.001$ uncorrected and rendered on a study-specific T1-weighted MRI template in MNI space. Analyses were adjusted for age, gender, total intracranial volume, and scanner type. The color bar indicates the Z-scores.

anterior insula, left thalamus, frontal lobe, temporal lobe, and the cerebellum when correcting for multiple comparisons (Supplementary Table 5). In the C9orf72 group, a significant negative correlation was found in the left middle frontal gyrus (Supplementary Table 6). In the MAPT group, the auditory hallucinations score was related to GM atrophy in the right angular gyrus (Supplementary Table 7). Areas related to tactile hallucinations (Fig. 3) in the GRN group (Supplementary Table 8) were similar to those seen in auditory hallucinations. In the C9orf72 group, we found a significant negative correlation in the inferior frontal gyrus and posterior cingulate gyrus (Supplementary Table 9). Significant areas were seen in the $M A P T$ group within the frontal lobe and left lingual gyrus (Supplementary Table 10).

As displayed in Fig. 4, GRN mutation carriers showed a significant association between the delusions score and GM atrophy mainly in the left thalamus and left anterior insula when correcting for multiple comparisons (Supplementary Table 11). In the C9orf72 group, we found a significant correlation between delusions and GM in the left middle and superior frontal gyri (Supplementary Table 12). In the MAPT group, the most significant areas for delusions were in the temporal lobe (left middle temporal gyrus and left hippocampus), frontal lobe, parietal lobe, left subcallosal area, left anterior insula, and fusiform gyrus when correcting for multiple comparisons (Supplementary Table 13).

For the depression score (Fig. 5), we found significant correlations within the GRN group, with GM in the frontal lobe, right subcallosal area, bilateral anterior insula, left middle cingulate, right cerebellum, temporal lobe, and right middle occipital 

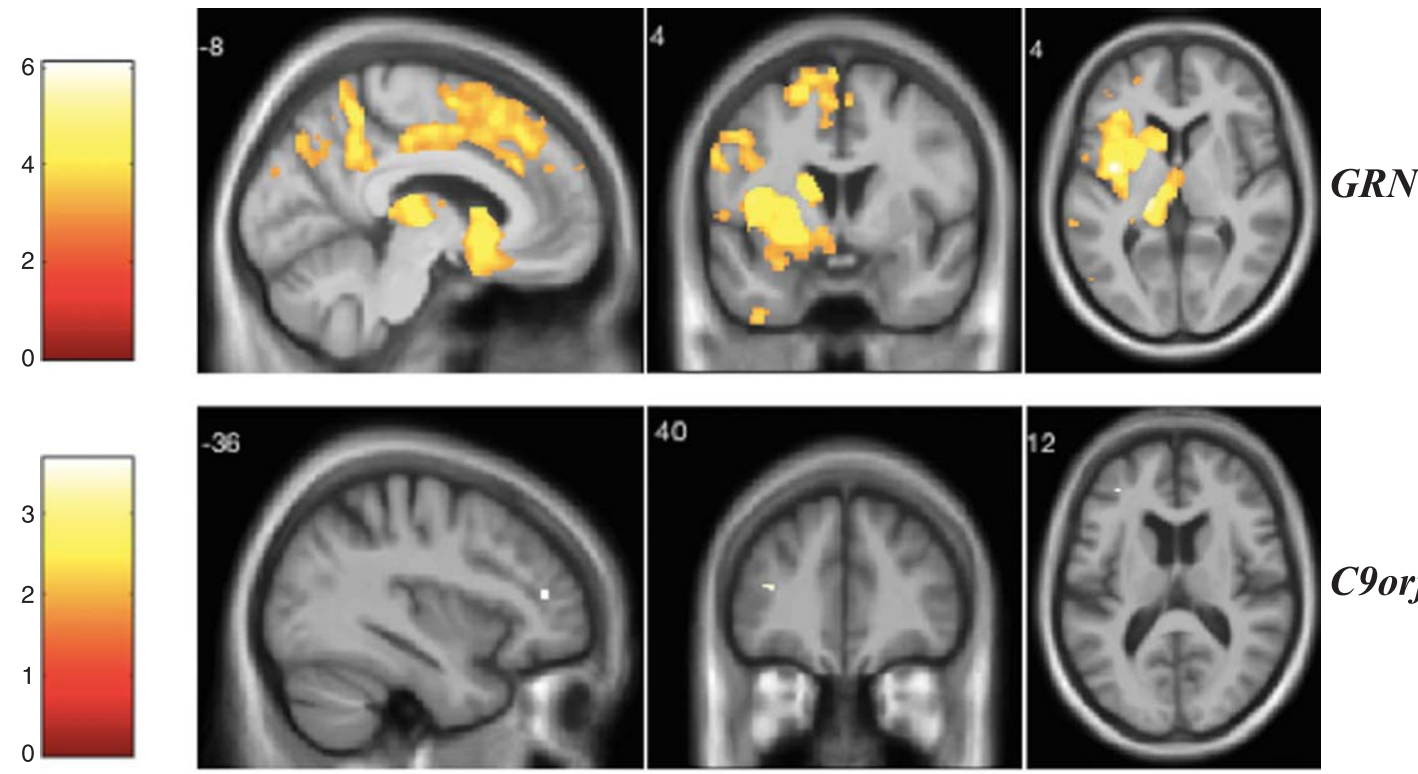

C9orf72
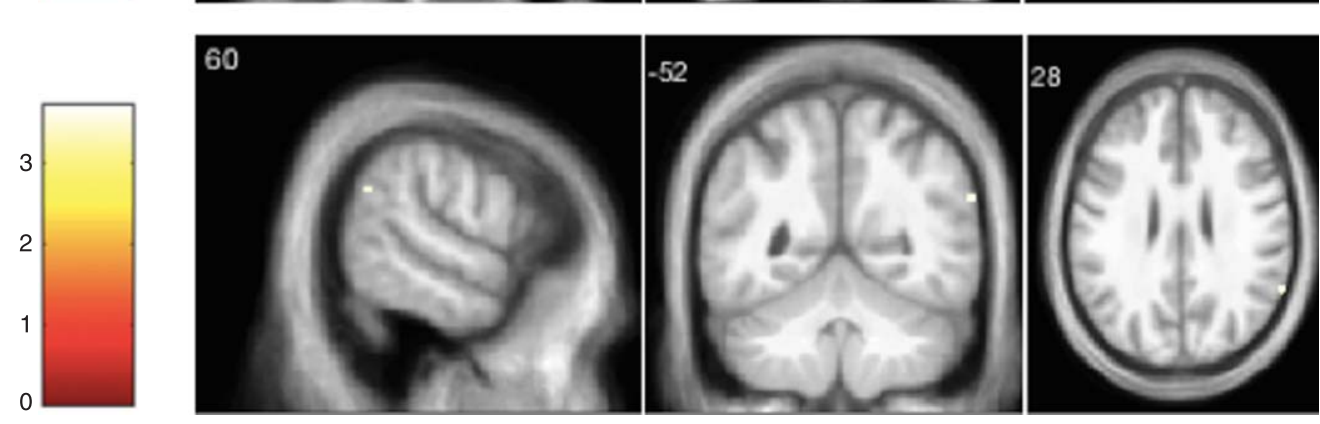

$M A P T$

Fig. 2. VBM analysis showing areas of significant correlation between the presence and severity of auditory hallucinations and GM density across the FTD genetic groups. Statistical parametric maps were thresholded at $p<0.001$ uncorrected and rendered on a study-specific T1-weighted MRI template in MNI space. Analyses were adjusted for age, gender, total intracranial volume, and scanner type. The color bar indicates the Z-scores.

gyrus (these clusters survived after FDR correction) (Supplementary Table 14). In C9orf72 expansion carriers, the depression score was correlated with GM atrophy in the parietal lobe (left precuneus and superior parietal lobule) and left superior frontal gyrus (Supplementary Table 15). In the MAPT group, we identified significant negative correlations with GM in the left postcentral gyrus, temporal lobe, right fusiform gyrus, right lingual gyrus, superior motor cortex, and left cerebellum (Supplementary Table 16).

Finally, for the anxiety score (Fig. 6), GRN mutation carriers showed significant correlations with GM atrophy in areas including the frontal lobe, left anterior insula, left precuneus, left posterior cingulate, temporal lobe, left caudate, left superior occipital gyrus, and bilateral cerebellum when correcting for multiple comparisons (Supplementary Table 17). In the C9orf72 group, we found significant correlations in the inferior frontal gyrus, left cerebellum, left fusiform gyrus and right superior temporal gyrus (Supplementary Table 18). In the MAPT group, the main significant areas were in the temporo-parietal and frontal lobes as well as bilateral cuneus (Supplementary Table 19).

Table 3 summarizes the regional grey matter correlates of NPS from the VBM analysis.

\section{DISCUSSION}

Our findings highlight the involvement of widespread cortical and subcortical brain structures, as part of large-scale networks, in the occurrence of NPS in FTD mutation carriers. These results suggest a strong association of psychotic symptoms with GM atrophy in the anterior insula in GRN mutation 


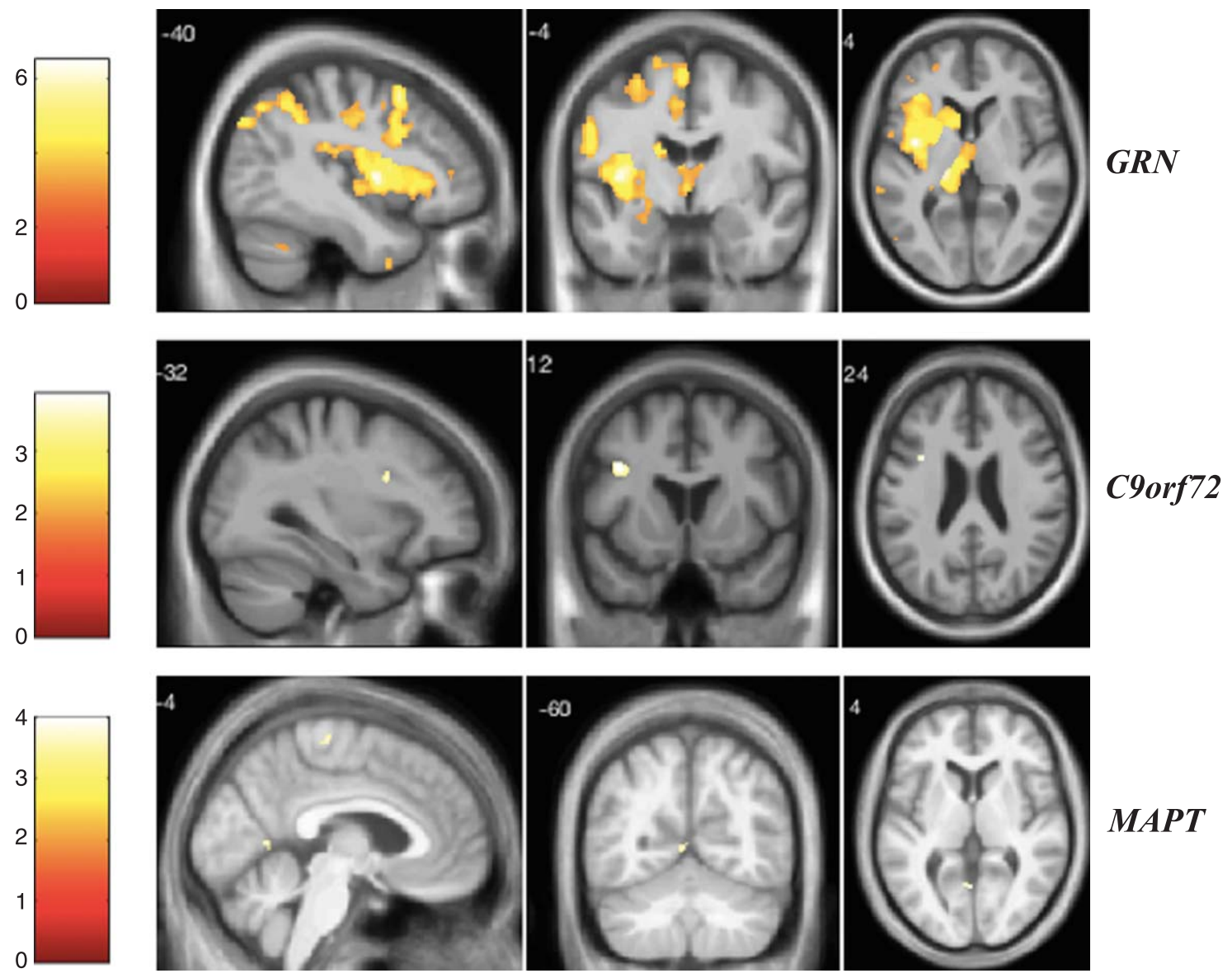

Fig. 3. VBM analysis showing areas of significant correlation between the presence and severity of tactile hallucinations and GM density across the FTD genetic groups. Statistical parametric maps were thresholded at $p<0.001$ uncorrected and rendered on a study-specific T1-weighted MRI template in MNI space. Analyses were adjusted for age, gender, total intracranial volume, and scanner type. The color bar indicates the Z-scores.

carriers and, to a lesser extent, in MAPT carriers. Moreover, the GRN group showed particular involvement of the left thalamus, left middle frontal gyrus, and cerebellum in the emergence of psychosis. In the $C 9$ orf 72 group, psychotic symptoms were mainly associated with changes in the frontal cortex, whereas the MAPT group involved the temporal lobe and angular gyri. The presence of mood disorders was mostly associated with GM atrophy in frontal-insular cortex, in addition to precuneus and posterior cingulate, in the $G R N$ group. Parietal and frontal cortices as well as cerebellum alterations correlated with mood disorders in $C 9$ orf 72 carriers, whereas in MAPT carriers, depression and anxiety were mainly associated with the temporo-parietal cortex.

The involvement of the insula in the emergence of psychotic symptoms in GRN mutations carriers supports the aberrant salience hypothesis previously reported in mental disorders [20,21]. The insula is critical for the regulation of a number of affective and cognitive functions, including body-self consciousness and subjective emotional experience. It is thought to integrate multisensory inputs and coordinate the allocation of the brain's attentional resources to the most relevant stimuli [22]. The anterior part of the insula, which is especially correlated to NPS in our study, was shown to be particularly specialized for interoception, subjective feelings, and emotional awareness [23]. Psychotic symptoms including delusions and all sensory modalities of hallucinations were also significantly associated with greater GM atrophy in the left thalamus within the GRN group. The disturbances of thalamo-cortical networks were suggested to contribute to the emergence of psychotic symptoms in schizophrenia [24].

The involvement of large-scale networks in the emergence of NPS in FTD is also reflected by the significant association of NPS with cerebellar 


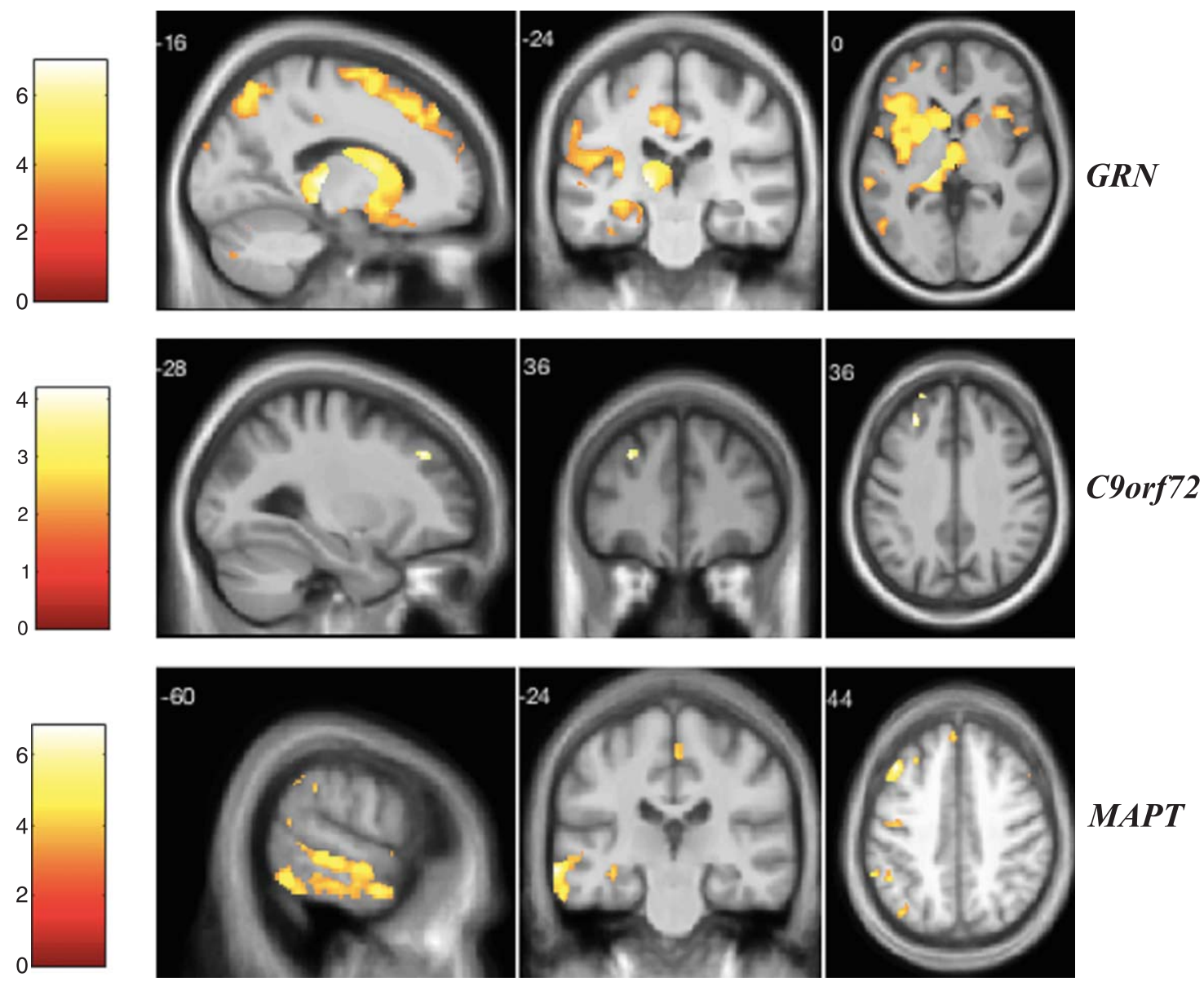

Fig. 4. VBM analysis showing areas of significant correlation between the presence and severity of delusions and GM density across the FTD genetic groups. Statistical parametric maps were thresholded at $p<0.001$ uncorrected and rendered on a study-specific T1-weighted MRI template in MNI space. Analyses were adjusted for age, gender, total intracranial volume, and scanner type. The color bar indicates the Z-scores.

alteration in the GRN group. A growing body of evidence arising from functional magnetic resonance imaging (fMRI) implicates cortico-cerebellar connectivity loops in the pathophysiology of primary psychiatric conditions [25]. Schmahmann et al. considered the psychosis spectrum as an integral part of the cerebellar cognitive affective syndrome and linked it to damage of the fastigial nucleus and the vermis, defined as "the limbic cerebellum" [26, 27]. Our results showed evidence of frontal involvement in delusions among C9orf72 expansion carriers which is inconsistent with a previous study suggesting an association between delusions and GM atrophy in the precuneus [28]. Limited data are available on the specific content of delusional thoughts in C9orf72 carriers. Authors have reported odd somatic complaints, somatoform delusions and body image distortion $[11,29]$. This observation is supported by the evidence of altered body scheme perception and processing among patients with C9orf72 expansion [30]. Interestingly, the superior and middle frontal gyri along with inferior frontal and posterior cingulate gyri, which correlated respectively with delusions and tactile hallucinations in our C9orf 72 group, have previously been considered as part of the neural correlates of somatoform disorders [31]. The MAPT group was mainly distinguished by the involvement of the left temporal lobe in delusional symptoms, in line with previous reports suggesting a predominant temporal alteration in MAPT mutation carriers $[12,32]$. This is also consistent with previous studies that reported an association between GM atrophy in temporo-limbic areas and vulnerability to psychosis [33].

As expected, visual hallucinations were mainly characterized by the involvement of the visual cortex 

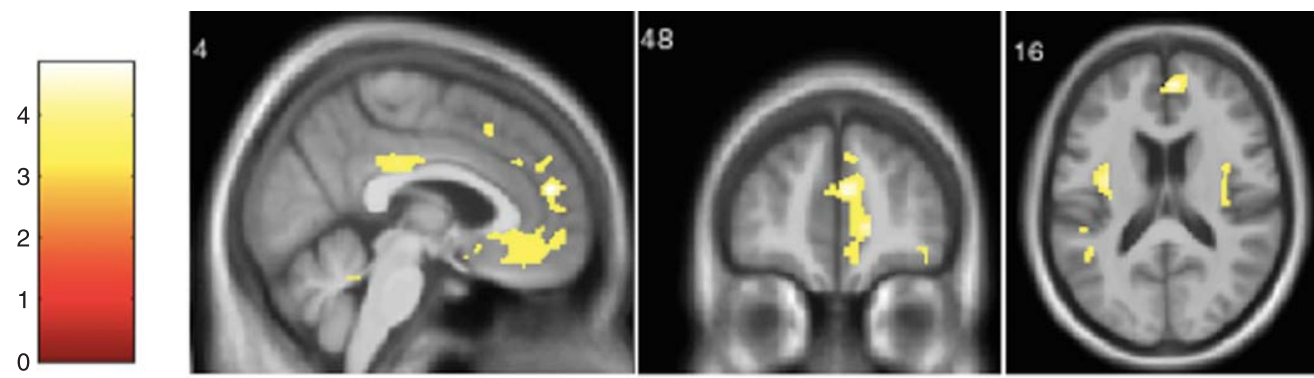

GRN
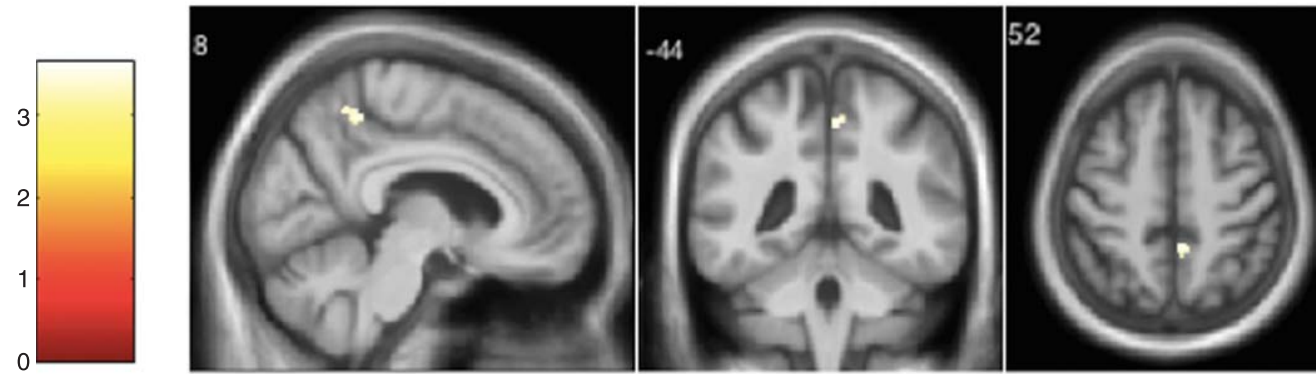

C9orf72
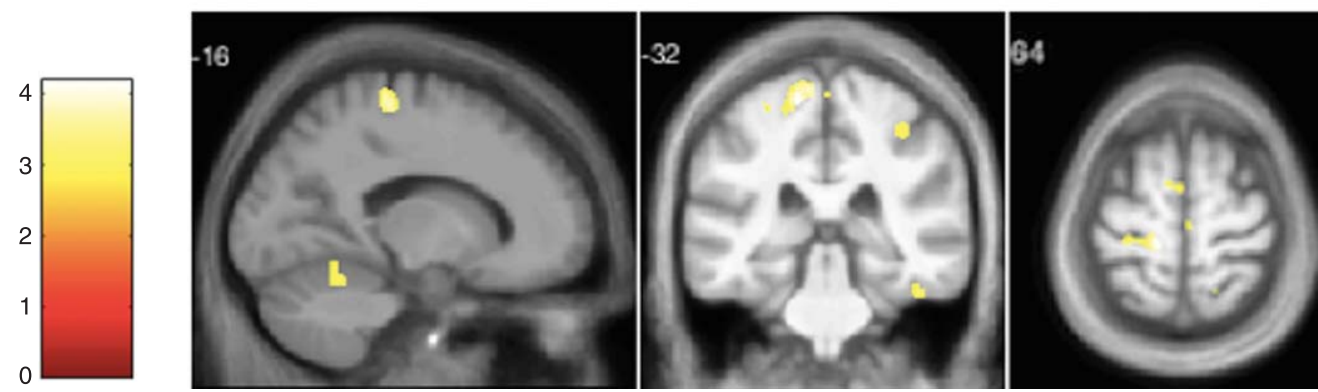

MAPT

Fig. 5. VBM analysis showing areas of significant correlation between the presence and severity of depression and GM density across the FTD genetic groups. Statistical parametric maps were thresholded at $p<0.001$ uncorrected and rendered on a study-specific T1-weighted MRI template in MNI space. Analyses were adjusted for age, gender, total intracranial volume, and scanner type. The color bar indicates the Z-scores.

including the occipito-parietal and occipito-temporal regions across the three genetic mutations, consistent with previous studies which demonstrated structural and functional disconnectivity within visual processing areas in hallucinatory experiences [34]. The correlation of visual hallucinations with GM in the left angular gyrus was shared by all genetic groups. The left angular gyrus alteration has previously been considered as a structural substrate in schizophrenia [35], but its role in the emergence of visual hallucinations remains unexplored. Based on our results, we might speculate that the angular gyrus, as part of the heteromodal parietal association cortex, plays presumably an important role in the psychopathology of visual hallucinations in genetic FTD. The GRN group was characterized by a significant correlation between thalamic atrophy, particularly posteriorly (including the pulvinar) and visual hallucinations.
Vulnerability to visual hallucinations as a result of thalamic dysfunction has been reported in neurodegenerative diseases such as Lewy body dementia (LBD) [36]. Interestingly visual hallucinations have previously been reported as an important distinctive feature in GRN carriers which could sometimes even present with a LBD-like phenotype [10]. Atrophy of the frontal lobe has also been found to correlate with visual hallucinations in both GRN and MAPT groups. Several studies have shown visual hallucinations to be linked to frontal structures, as reflected in previous correlational analyses performed in LBD and Parkinson's disease [37].

Mood disorders in the GRN group were mainly related to a fronto-insular-cerebellar alteration. We also noted particular involvement of the posterior portions of the default-mode network (DMN) (precuneus and posterior cingulate cortex) in anxiety and 

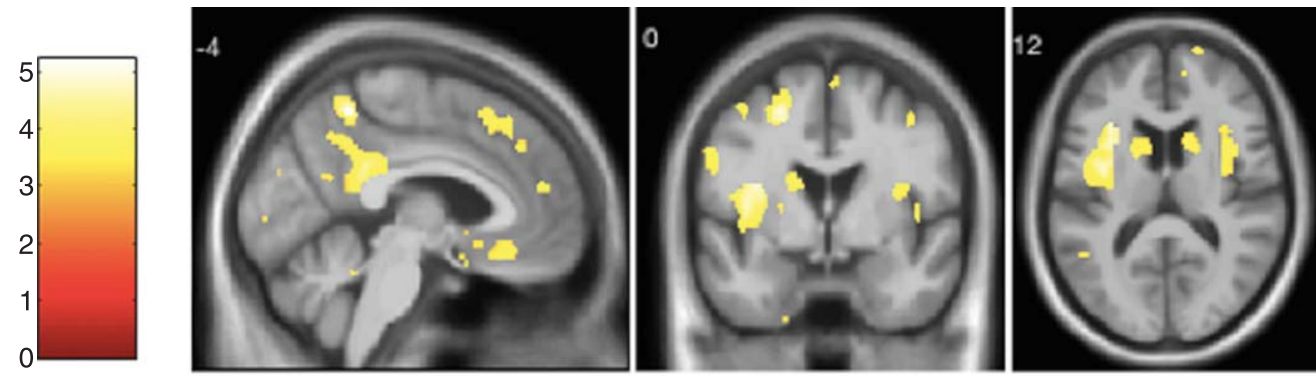

$G R N$
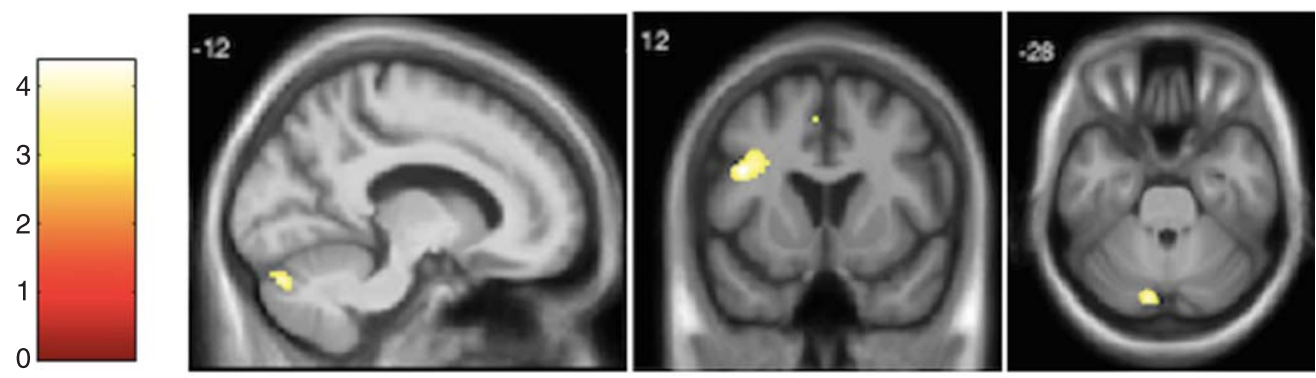

C9orf72
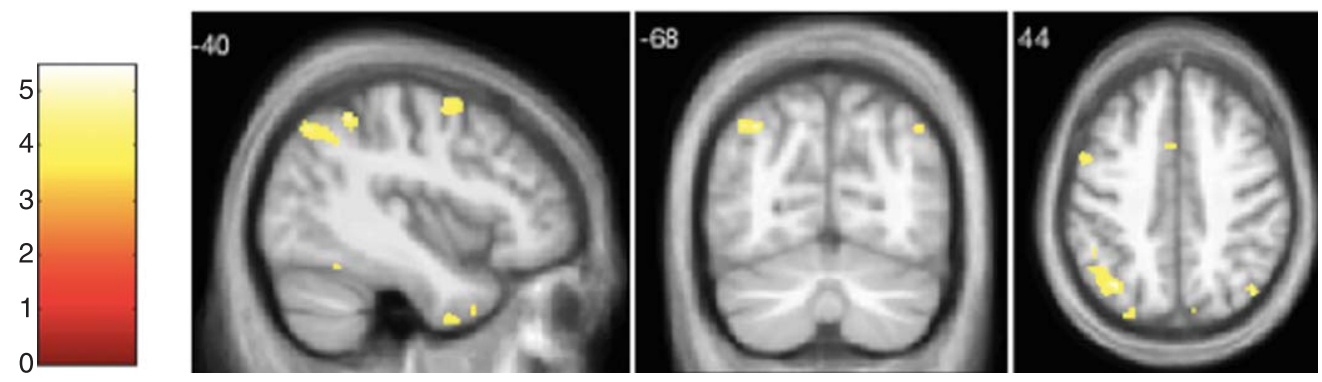

MAPT

Fig. 6. VBM analysis showing areas of significant correlation between the presence and severity of anxiety and GM density across the FTD genetic groups. Statistical parametric maps were thresholded at $p<0.001$ uncorrected and rendered on a study-specific T1-weighted MRI template in MNI space. Analyses were adjusted for age, gender, total intracranial volume, and scanner type. The color bar indicates the Z-scores.

of left hippocampus in both anxiety and depression. Left precuneus atrophy has also been found to be correlated to depression in C9orf72 expansion carriers. This study supports previous findings of the role of DMN alterations in depression and anxiety disorders [38-40]. The MAPT group was characterized by the involvement of right temporo-occipital areas in depression and left temporo-parietal regions in anxiety. This is in line with the prominence of mood disorders in neurodegenerative disease presenting with a major alteration of posterior cortical areas as illustrated by posterior cortical atrophy [41, 42]. Disparate findings for depression could be reconciled through its conceptualization as a constellation of symptoms related to several psychopathological mechanisms (self-related mental representation, referential judgments, negative selffocused thoughts...), which can be underpinned by different structural and neurobiological dysfunctions [43, 44]. Though the structural models underlying the psychopathology of depression can be distinct, they may involve overlapping functional networks and neurobiological systems including serotoninergic, noradrenergic and dopaminergic tracts.

Anxiety was previously reported as a prominent feature in $C 9$ orf 72 expansion carriers [45]. It is interesting to note that, despite a recognized pattern of cerebellar involvement in the C9orf72 expansion [46], its atrophy has proved to be only related to anxiety in our study, but not to psychosis as previously reported [47]. Variability in findings between studies may be due to several factors, including sample size, different neuroimaging techniques, and medication. Overall, we found the most significant results in the GRN group. 


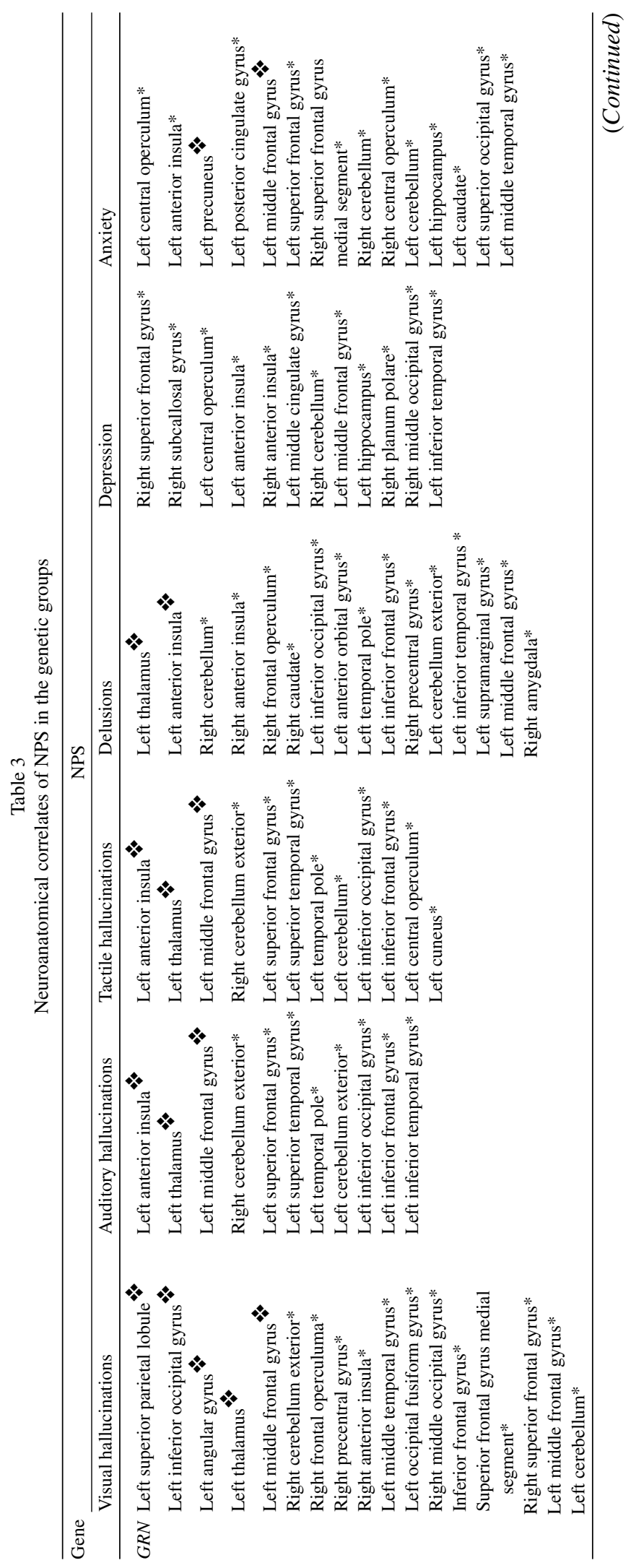




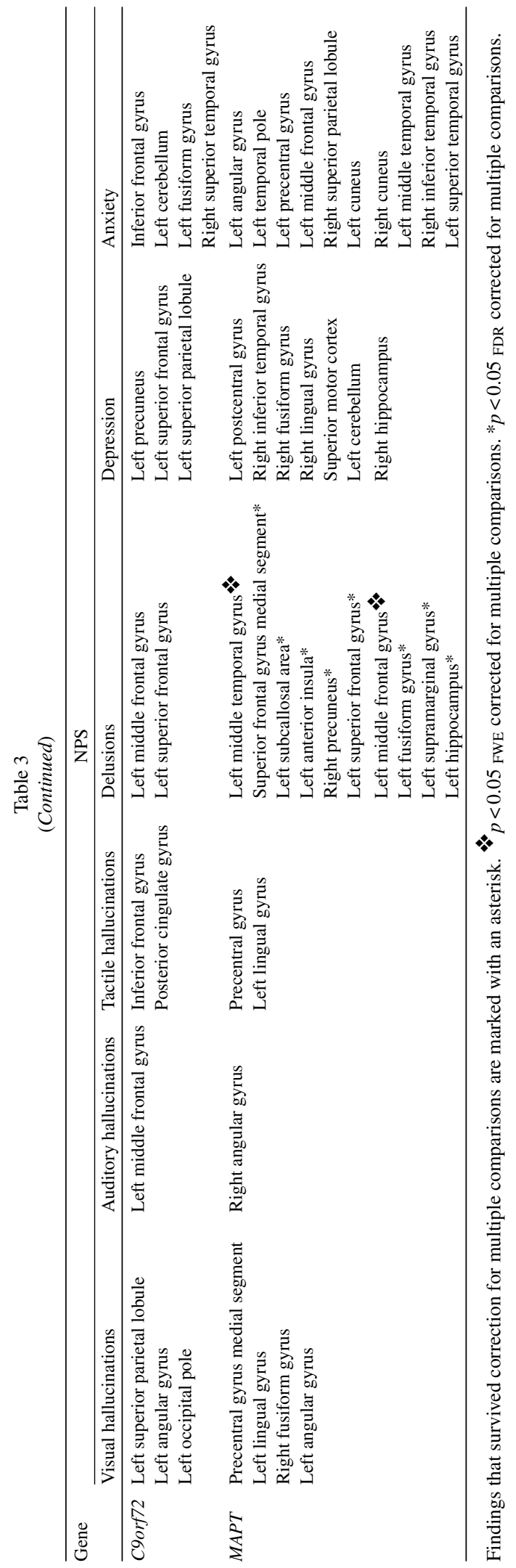


The lack of specific identification of each symptom's nature through the use of the NPS scale instead of a full psychiatric questionnaire is a limitation. Psychotic symptoms are a multidimensional phenomenon, which is difficult to deconstruct through a clinical scale, therefore caution should be taken in interpreting the results. For instance, odd somatic complaints in C9orf 72 expansion carriers may be mistaken for anxiety disorders. Further studies should be performed based on detailed psychiatric assessment, including expanded items of valid psychiatric rating scales, to improve the detection of subtle NPS and better refine their phenomenology. Connectivitybased neuroanatomical methods will be required in order to provide a better understanding of the underlying structural substrates and networks dynamics of psychotic symptoms.

Despite these limitations, to our knowledge, this is the first study to investigate the neuroanatomical correlates of a wide range of NPS in the three main mutations responsible for genetic FTD. Moreover, our findings, taking into account the presymptomatic phase of the disease, support that NPS may be considered as prodromal hallmarks of early neurodegeneration, within the scope of mild behavioral impairment (MBI). The concept of MBI was developed as a potential pre-dementia stage. New diagnostic criteria and a standardized scale to measure MBI have recently emerged [48, 49]. Affective dysregulation and abnormal perception/thought content were stated as MBI subcategories. A previous study showed that MBI patients had a high conversion rate to dementia $(71.5 \%)$, mainly evolvement to FTD [50]. Therefore, the study of NPS offers the opportunity of early diagnosis and could allow measurement of future disease-modifying preventive strategies in at-risk individuals.

\section{Conclusions}

Our findings demonstrated that structural correlates of NPS may be distinct in the three main forms of genetic FTD, which could theoretically explain the differences observed in neuropsychiatric phenomenology between genetic subtypes. Additionally, our study supported that clinical overlap between FTD and primary psychiatric conditions may be mediated by the alteration of common structures involved in large-scale networks. Future studies should consider detailed neuropsychiatric assessment and perform a multimodal neuroimaging approach to further explore the functional networks and neural substrates of specific NPS.

\section{ACKNOWLEDGMENTS}

The authors are grateful to the participants and their families for supporting our research. We wish to thank Audrey Paradis, Marie-Claire Doré, Nancy Parent, Mélanie Harvey, and the MRI team led by Mylène Jalbert at Hôpital de l'Enfant Jésus in Québec. We wish also to thank the members of the Dementia Research Centre at UCL.

This work was supported by the Institut de recherche en santé du Canada (IRSC), Weston Brain Institute, Fondation du Centre hospitalier Universitaire de Québec, Centre d'intégration de la recherche et des technologies sur les maladies cognitives (CIRTMC), Société Alzheimer de Québec and Départements de médecine, de psychiatrie et de neurosciences de l'Université Laval.

The Dementia Research Centre was supported by Alzheimer's Research UK, Brain Research Trust, and The Wolfson Foundation. This work was supported by the NIHR Queen Square Dementia Biomedical Research Unit, the NIHR UCL/H Biomedical Research Centre and the Leonard Wolfson Experimental Neurology Centre (LWENC) Clinical Research Facility. This work was supported by the Bluefield Project and the MRC UK GENFI grant (MR/M023664/1).

Jonathan D. Rohrer was supported by an MRC Clinician Scientist Fellowship (MR/M008525/1) and has received funding from the NIHR Rare Disease Translational Research Collaboration (BRC149/NS/MH). Jason D. Warren was supported by the Alzheimer's Society and the NIHR UCLH Biomedical Research Centre.

This work was also funded by the UK Medical Research Council, the Italian Ministry of Health and the Canadian Institutes of Health Research as part of a Centres of Excellence in Neurodegeneration grant, and also a Canadian Institutes of Health Research operating grant and funding from the Weston Brain Institute to Mario Masselis.

Authors' disclosures available online (https:// www.j-alz.com/manuscript-disclosures/18-0053r3).

\section{SUPPLEMENTARY MATERIAL}

The supplementary material is available in the electronic version of this article: http://dx.doi.org/ 10.3233/JAD-180053. 


\section{REFERENCES}

[1] Seelaar H, Rohrer JD, Pijnenburg YA, Fox NC, van Swieten JC (2011) Clinical, genetic and pathological heterogeneity of frontotemporal dementia: A review. J Neurol Neurosurg Psychiatry 82, 476-486.

[2] Rohrer JD, Guerreiro R, Vandrovcova J, Uphill J, Reiman D, Beck J, Isaacs AM, Authier A, Ferrari R, Fox NC, Mackenzie IR, Warren JD, de Silva R, Holton J, Revesz T, Hardy J, Mead S, Rossor MN (2009) The heritability and genetics of frontotemporal lobar degeneration. Neurology 73, 14511456.

[3] Rohrer JD, Warren JD (2011) Phenotypic signatures of genetic frontotemporal dementia. Curr Opin Neurol 24, $542-549$.

[4] Woolley JD, Khan BK, Murthy NK, Miller BL, Rankin KP (2011) The diagnostic challenge of psychiatric symptoms in neurodegenerative disease: Rates of and risk factors for prior psychiatric diagnosis in patients with early neurodegenerative disease. J Clin Psychiatry 72, 126-133.

[5] Rascovsky K, Hodges JR, Knopman D, Mendez MF, Kramer JH, Neuhaus J (2011) Sensitivity of revised diagnostic criteria for the behavioural variant of frontotemporal dementia. Brain 134(Pt 9), 2456-2477.

[6] Landqvist Waldo M, Gustafson L, Passant U, Englund E (2015) Psychotic symptoms in frontotemporal dementia: A diagnostic dilemma? Int Psychogeriatr 27, 531-539.

[7] Galimberti D, Dell' Osso B, Altamura AC, Scarpini E (2015) Psychiatric symptoms in frontotemporal dementia: Epidemiology, phenotypes, and differential diagnosis. Biol Psychiatry 78, 684-692.

[8] Snowden JS, Adams J, Harris J, Thompson JC, Rollinson S, Richardson A, Jones M, Neary D, Mann DM, Pickering-Brown S (2015) Distinct clinical and pathological phenotypes in frontotemporal dementia associated with MAPT, PGRN and C9orf72 mutations. Amyotroph Lateral Scler Frontotemporal Degener 16, 497-505.

[9] Shinagawa S, Nakajima S, Plitman E, Graff-Guerrero A, Mimura M, Nakayama K, Miller BL (2014) Psychosis in frontotemporal dementia. J Alzheimers Dis 42, 485-499.

[10] Le Ber I, Camuzat A, Hannequin D, Pasquier F, Guedj E, Rovelet-Lecrux A, Hahn-Barma V, van der Zee J, Clot F, Bakchine S, Puel M, Ghanim M, Lacomblez L, Mikol J, Deramecourt V, Lejeune P, de la Sayette V, Belliard S, Vercelletto M, Meyrignac C, Van Broeckhoven C, Lambert JC, Verpillat P, Campion D, Habert MO, Dubois B, Brice A (2008) Phenotype variability in progranulin mutation carriers: A clinical, neuropsychological, imaging and genetic study. Brain 131, 732-746.

[11] Ducharme S, Bajestan S, Dickerson BC, Voon V (2017) Psychiatric presentations of C9orf72 mutation: What are the diagnostic implications for clinicians? J Neuropsychiatry Clin Neurosci 29, 195-205.

[12] Rohrer JD, Nicholas JM, Cash DM, van Swieten J, Dopper E, Jiskoot L, van Minkelen R, Rombouts SA, Cardoso MJ, Clegg S, Espak M, Mead S, Thomas DL, De Vita E, Masellis M, Black SE, Freedman M, Keren R, MacIntosh BJ, Rogaeva E, Tang-Wai D, Tartaglia MC, Laforce R Jr, Tagliavini F, Tiraboschi P, Redaelli V, Prioni S, Grisoli M, Borroni B, Padovani A, Galimberti D, Scarpini E, Arighi A, Fumagalli G, Rowe JB, Coyle-Gilchrist I, Graff C, Fallström M, Jelic V, Ståhlbom AK, Andersson C, Thonberg H, Lilius L, Frisoni GB, Pievani M, Bocchetta M, Benussi L, Ghidoni R, Finger E, Sorbi S, Nacmias B, Lombardi G, Polito C, Warren JD, Ourselin S, Fox NC, Rossor MN (2015)
Presymptomatic cognitive and neuroanatomical changes in genetic frontotemporal dementia in the Genetic Frontotemporal dementia Initiative (GENFI) study: A cross-sectional analysis. Lancet Neurol 14, 253-262.

[13] Cash DM, Bocchetta M, Thomas DL, Dick KM, van Swieten JC, Borroni B, Galimberti D, Masellis M, Tartaglia MC, Rowe JB, Graff C, Tagliavini F, Frisoni GB, Laforce R Jr, Finger E, de Mendonca A, Sorbi S, Rossor MN, Ourselin S, Rohrer JD, Genetic FTD Initiative, GENFI (2018) Patterns of gray matter atrophy in genetic frontotemporal dementia: Results from the GENFI study. Neurobiol Aging 62, 191-196.

[14] Palaniyappan L, Liddle PF (2012) Does the salience network play a cardinal role in psychosis? An emerging hypothesis of insular dysfunction. J Psychiatry Neurosci 37, 17-27.

[15] Cummings JL (1997) The Neuropsychiatric Inventory: Assessing psychopathology in dementia patients. Neurology 48, S10-16.

[16] Ashburner J (2007) A fast diffeomorphic image registration algorithm. Neuroimage 38, 95-113.

[17] Ridgway GR, Omar R, Ourselin S, Hill DL, Warren JD, Fox NC (2009) Issues with threshold masking in voxel-based morphometry of atrophied brains. Neuroimage 44, 99-111.

[18] Malone IB, Leung KK, Clegg S, Barnes J, Whitwell JL, Ashburner J, Fox NC, Ridgway GR (2015) Accurate automatic estimation of total intracranial volume: A nuisance variable with less nuisance. Neuroimage 104, 366-372.

[19] Mioshi E, Hsieh S, Savage S, Hornberger M, Hodges JR (2010) Clinical staging and disease progression in frontotemporal dementia. Neurology 74, 1591-1597.

[20] Wylie KP, Tregellas JR (2010) The role of the insula in schizophrenia. Schizophr Res 123, 93-104.

[21] Gaebler AJ, Mathiak K, Koten JW Jr, Konig AA, Koush Y, Weyer D, Depner C, Matentzoglu S, Edgar JC, Willmes K, Zvyagintsev M (2015) Auditory mismatch impairments are characterized by core neural dysfunctions in schizophrenia. Brain 138, 1410-1423.

[22] Seeley WW, Menon V, Schatzberg AF, Keller J, Glover GH, Kenna H, Reiss AL, Greicius MD (2007) Dissociable intrinsic connectivity networks for salience processing and executive control. $J$ Neurosci 27, 2349-2356.

[23] Craig AD (2009) How do you feel-now? The anterior insula and human awareness. Nat Rev Neurosci 10, 59-70.

[24] Anticevic A, Cole MW, Repovs G, Murray JD, Brumbaugh MS, Winkler AM, Savic A, Krystal JH, Pearlson GD, Glahn DC (2014) Characterizing thalamo-cortical disturbances in schizophrenia and bipolar illness. Cereb Cortex 24, 31163130.

[25] Chen YL, Tu PC, Lee YC, Chen YS, Li CT, Su TP (2013) Resting-state fMRI mapping of cerebellar functional dysconnections involving multiple large-scale networks in patients with schizophrenia. Schizophr Res 149, 26-34.

[26] Schmahmann JD, Weilburg JB, Sherman JC (2007) The neuropsychiatry of the cerebellum - insights from the clinic. Cerebellum 6, 254-267.

[27] Laforce R Jr, Buteau JP, Bouchard JP, Rouleau GA, Bouchard RW, Dupre N (2010) Cognitive impairment in ARCA-1, a newly discovered pure cerebellar ataxia syndrome. Cerebellum 9, 443-453.

[28] Shinagawa S, Naasan G, Karydas AM, Coppola G, Pribadi M, Seeley WW, Trojanowski JQ, Miller BL, Grinberg LT (2015) Clinicopathological study of patients with C9ORF72-associated frontotemporal dementia presenting with delusions. J Geriatr Psychiatry Neurol 28, 99-107. 
[29] Snowden JS, Rollinson S, Thompson JC, Harris JM, Stopford CL, Richardson AM, Jones M, Gerhard A, Davidson YS, Robinson A, Gibbons L, Hu Q, DuPlessis D, Neary D, Mann DM, Pickering-Brown SM (2012) Distinct clinical and pathological characteristics of frontotemporal dementia associated with C9ORF72 mutations. Brain 135, 693-708.

[30] Downey LE, Fletcher PD, Golden HL, Mahoney CJ, Agustus JL, Schott JM, Rohrer JD, Beck J, Mead S, Rossor MN, Crutch SJ, Warren JD (2014) Altered body schema processing in frontotemporal dementia with C9ORF72 mutations. J Neurol Neurosurg Psychiatry 85, 1016-1023.

[31] Boeckle M, Schrimpf M, Liegl G, Pieh C (2016) Neural correlates of somatoform disorders from a meta-analytic perspective on neuroimaging studies. Neuroimage Clin 11, 606-613.

[32] Whitwell JL, Weigand SD, Boeve BF, Senjem ML, Gunter JL, DeJesus-Hernandez M, Rutherford NJ, Baker M, Knopman DS, Wszolek ZK, Parisi JE, Dickson DW, Petersen RC, Rademakers R, Jack CR, Jr, Josephs KA (2012) Neuroimaging signatures of frontotemporal dementia genetics: C9ORF72, tau, progranulin and sporadics. Brain 135, 794806.

[33] Fusar-Poli P, Radua J, McGuire P, Borgwardt S (2012) Neuroanatomical maps of psychosis onset: Voxelwise meta-analysis of antipsychotic-naive VBM studies. Schizophr Bull 38, 1297-1307.

[34] Zmigrod L, Garrison JR, Carr J, Simons JS (2016) The neural mechanisms of hallucinations: A quantitative metaanalysis of neuroimaging studies. Neurosci Biobehav Rev 69, 113-123.

[35] Nierenberg J, Salisbury DF, Levitt JJ, David EA, McCarley RW, Shenton ME (2005) Reduced left angular gyrus volume in first-episode schizophrenia. Am J Psychiatry 162, 15391541.

[36] Delli Pizzi S, Maruotti V, Taylor JP, Franciotti R, Caulo M, Tartaro A, Thomas A, Onofrj M, Bonanni L (2014) Relevance of subcortical visual pathways disruption to visual symptoms in dementia with Lewy bodies. Cortex $\mathbf{5 9}$, 12-21.

[37] Sanchez-Castaneda C, Rene R, Ramirez-Ruiz B, Campdelacreu J, Gascon J, Falcon C, Calopa M, Jauma S, Juncadella M, Junque C (2010) Frontal and associative visual areas related to visual hallucinations in dementia with Lewy bodies and Parkinson's disease with dementia. Mov Disord 25, 615-622.

[38] Strawn JR, Wehry AM, Chu WJ, Adler CM, Eliassen JC, Cerullo MA, Strakowski SM, Delbello MP (2013) Neuroanatomic abnormalities in adolescents with generalized anxiety disorder: A voxel-based morphometry study. Depress Anxiety 30, 842-848.

[39] Brakowski J, Spinelli S, Dorig N, Bosch OG, Manoliu A, Holtforth MG, Seifritz E (2017) Resting state brain network function in major depression - Depression symptomatology, antidepressant treatment effects, future research. J Psychiatr Res 92, 147-159.
[40] Cavanna AE, Trimble MR (2006) The precuneus: A review of its functional anatomy and behavioural correlates. Brain 129, 564-583.

[41] Wolf RC, SchöNfeldt-Lecuona C (2006) Depressive symptoms as first manifestation of posterior cortical atrophy. Am J Psychiatry 163, 939-940.

[42] Mendez MF, Ghajarania M, Perryman KM (2002) Posterior cortical atrophy: Clinical characteristics and differences compared to Alzheimer's disease. Dement Geriatr Cogn Disord 14, 33-40.

[43] Coutinho JF, Fernandesl SV, Soares JM, Maia L, Goncalves OF, Sampaio A (2016) Default mode network dissociation in depressive and anxiety states. Brain Imaging Behav 10, 147-157.

[44] Philippi CL, Cornejo MD, Frost CP, Walsh EC, Hoks RM, Birn R, Abercrombie HC (2018) Neural and behavioral correlates of negative self-focused thought associated with depression. Hum Brain Mapp 39, 2246-2257.

[45] Mahoney CJ, Beck J, Rohrer JD, Lashley T, Mok K, Shakespeare T, Yeatman T, Warrington EK, Schott JM, Fox NC, Rossor MN, Hardy J, Collinge J, Revesz T, Mead S, Warren JD (2012) Frontotemporal dementia with the C9ORF72 hexanucleotide repeat expansion: Clinical, neuroanatomical and neuropathological features. Brain 135, 736-750.

[46] Bocchetta M, Cardoso MJ, Cash DM, Ourselin S, Warren JD, Rohrer JD (2016) Patterns of regional cerebellar atrophy in genetic frontotemporal dementia. Neuroimage Clin 11, 287-290.

[47] Devenney EM, Landin-Romero R, Irish M, Hornberger M, Mioshi E, Halliday GM, Kiernan MC, Hodges JR (2017) The neural correlates and clinical characteristics of psychosis in the frontotemporal dementia continuum and the C9orf72 expansion. Neuroimage Clin 13, 439-445.

[48] Ismail Z, Smith EE, Geda Y, Sultzer D, Brodaty H, Smith G, Aguera-Ortiz L, Sweet R, Miller D, Lyketsos CG, Area INSPI (2016) Neuropsychiatric symptoms as early manifestations of emergent dementia: Provisional diagnostic criteria for mild behavioral impairment. Alzheimers Dement 12, 195-202.

[49] Ismail Z, Aguera-Ortiz L, Brodaty H, Cieslak A, Cummings J, Fischer CE, Gauthier S, Geda YE, Herrmann N, Kanji J, Lanctot KL, Miller DS, Mortby ME, Onyike CU, Rosenberg PB, Smith EE, Smith GS, Sultzer DL, Lyketsos C, NPS Professional Interest Area of the International Society of to Advance Alzheimer's Research and Treatment (NPS-PIA of ISTAART) (2017) The Mild Behavioral Impairment Checklist (MBI-C): A rating scale for neuropsychiatric symptoms in pre-dementia populations. J Alzheimers Dis 56, 929-938.

[50] Taragano FE, Allegri RF, Heisecke SL, Martelli MI, Feldman ML, Sanchez V, Garcia VA, Tufro G, Castro DM, Leguizamon PP, Guelar V, Ruotolo E, Zegarra C, Dillon C (2018) Risk of conversion to dementia in a mild behavioral impairment group compared to a psychiatric group and to a mild cognitive impairment group. J Alzheimers Dis $\mathbf{6 2}$, 227-238. 


\section{APPENDIX: LIST OF CONSORTIUM MEMBERS}

Christin Andersson - Department of Clinical Neuroscience, Karolinska Institutet, Stockholm, Sweden; [christin.andersson@karolinska.se]

Silvana Archetti - Biotechnology Laboratory, Department of Diagnostics, Civic Hospital of Brescia, Brescia, Italy; [archetti.s@ tiscali.it]

Luisa Benussi - Istituto di Ricovero e Cura a Carattere Scientifico Istituto Centro San Giovanni di Dio Fatebenefratelli, Brescia, Italy; [lbenussi@fatebenefratelli.eu]

Giuliano Binetti - Istituto di Ricovero e Cura a Carattere Scientifico Istituto Centro San Giovanni di Dio Fatebenefratelli, Brescia, Italy; [gbinetti@fatebenefratelli.eu]

Sandra Black - LC Campbell Cognitive Neurology Research Unit, Sunnybrook Research Institute, Toronto, Canada; [sandra.black@ sunnybrook.ca]

Maura Cosseddu - Centre of Brain Aging, University of Brescia, Brescia, Italy; [maura.cosseddu@gmail.com]

Marie Fallström - Department of Geriatric Medicine, Karolinska University Hospital, Stockholm, Sweden; [marie.fallstrom@karolinska.se]

Carlos Ferreira - Instituto Ciências Nucleares Aplicadas á Saúde, Universidade de Coimbra, Coimbra, Portugal [c_dferreira@yahoo.com]

Nick C Fox - Dementia Research Centre, UCL Institute of Neurology, UK; [n.fox@ ucl.ac.uk]

Morris Freedman - Division of Neurology, Baycrest Centre for Geriatric Care, University of Toronto, Canada; [mfreedman@baycrest.org]

Stefano Gazzina - Centre of Brain Aging, Neurology Unit, Department of Clinical and Experimental Sciences, University of Brescia, Brescia, Italy; [stefanogazzina@alice.it]

Roberta Ghidoni - Istituto di Ricovero e Cura a Carattere Scientifico Istituto Centro San Giovanni di Dio Fatebenefratelli, Brescia, Italy; [rghidoni@fatebenefratelli.eu]

Marina Grisoli - Fondazione Istituto di Ricovero e Cura a Carattere Scientifico Istituto Neurologico Carlo Besta, Milano, Italy; [Marina.Grisoli@ istitutobesta.it]

Vesna Jelic - Division of Clinical Geriatrics, Karolinska Institutet, Stockholm, Sweden; [vesna.jelic@ki.se]

Lize Jiskoot - Department of Neurology, Erasmus Medical Center, Rotterdam; [1.c.jiskoot@erasmusmc.nl]
Ron Keren - University Health Network Memory Clinic, Toronto Western Hospital, Toronto, Canada; [Ron.Keren@uhn.ca]

Gemma Lombardi - Department of Neuroscience, Psychology, Drug Research and Child Health, University of Florence, Florence, Italy; [gemmalomb@gmail.com]

Carolina Maruta - Lisbon Faculty of Medicine, Language Research Laboratory, Lisbon, Portugal; [carolmaruta@gmail.com]

Simon Mead - MRC Prion Unit, Department of Neurodegenerative Disease, UCL Institute of Neurology, Queen Square, London, UK; [s.mead@ prion.ucl.ac.uk]

Lieke Meeter - Department of Neurology, Erasmus Medical Center, Rotterdam, Netherlands; [h.meeter@erasmusmc.nl]

Rick van Minkelen - Department of Clinical Genetics, Erasmus Medical Center, Rotterdam, Netherlands; [r.vanminkelen@erasmusmc.nl]

Benedetta Nacmias - Department of Neuroscience, Psychology, Drug Research and Child Health, University of Florence, Florence, Italy; [benedetta.nacmias@unifi.it]

Linn öijerstedt - Division of Neurogeriatrics, Karolinska Institutet, Stockholm, Sweden; [linn.oijerstedt@ki.se]

Sebastien Ourselin - Centre for Medical Image Computing, University College London, UK; [s.ourselin@ucl.ac.uk]

Alessandro Padovani - Neurology Unit, Department of Medical and Experimental Sciences, University of Brescia, Brescia, Italy; [alessandro.padovani@unibs.it]

Jessica Panman - Department of Neurology, Erasmus Medical Center, Rotterdam, Netherlands; [j.panman@erasmusmc.nl]

Michela Pievani - Istituto di Ricovero e Cura a Carattere Scientifico Istituto Centro San Giovanni di Dio Fatebenefratelli, Brescia, Italy; [mpievani@fatebenefratelli.eu]

Cristina Polito - Department of Clinical Pathophysiology, University of Florence, Florence, Italy; [cristina.polito@unifi.it]

Enrico Premi - Centre for Ageing Brain and Neurodegenerative Disorders, Neurology Unit, University of Brescia, Brescia, Italy; [zedtower@gmail.com]

Sara Prioni - Fondazione Istituto di Ricovero e Cura a Carattere Scientifico Istituto Neurologico Carlo Besta, Milano, Italy; [Sara.Prioni@istitutobesta.it] 
Rosa Rademakers [as London Ontario geneticist] - Department of Neurosciences, Mayo Clinic, Jacksonville, Florida; [Rademakers.Rosa@mayo.edu]

Veronica Redaelli - Fondazione Istituto di Ricovero e Cura a Carattere Scientifico Istituto Neurologico Carlo Besta, Milano, Italy; [Veronica. Redaelli@istituto-besta.it]

Ekaterina Rogaeva - Tanz Centre for Research in Neurodegenerative Diseases, University of Toronto, Canada; [ekaterina.rogaeva@utoronto.ca]

Giacomina Rossi - Fondazione Istituto di Ricovero e Cura a Carattere Scientifico Istituto Neurologico Carlo Besta, Milano, Italy; [Giacomina. Rossi@istituto-besta.it]

Martin N Rossor - Dementia Research Centre, UCL Institute of Neurology, UK; [m.rossor@ ucl.ac.uk]

David Tang-Wai - University Health Network Memory Clinic, Toronto Western Hospital, Toronto, Canada; [David.Tang-Wai@uhn.ca]
David L Thomas - Neuroradiological Academic Unit, UCL Institute of Neurology, UK; [d.thomas@ucl.ac.uk]

Hakan Thonberg - Center for Alzheimer Research, Division of Neurogeriatrics, Karolinska Institutet, Stockholm, Sweden; [hakan.thonberg@ karolinska.se]

Pietro Tiraboschi - Fondazione Istituto di Ricovero e Cura a Carattere Scientifico Istituto Neurologico Carlo Besta, Milano, Italy; [Pietro.Tiraboschi@istituto-besta.it]

Ana Verdelho - Department of Neurosciences, Santa Maria Hospital, University of Lisbon, Portugal; [averdelho@medicina.ulisboa.pt]

Jason D Warren - Dementia Research Centre, UCL Institute of Neurology, UK; [jason.warren@ ucl.ac.uk] 